Report no. 95/13

\title{
Stability and accuracy of numerical boundary conditions in aeroelastic analysis
}

\author{
M. B. Giles \\ Oxford University Computing Laboratory \\ Numerical Analysis Group
}

This paper analyses the accuracy and numerical stability of coupling procedures in aeroelastic modelling. A two-dimensional model problem assuming unsteady inviscid flow past an oscillating wall leads to an even simpler one-dimensional model problem. Analysis of different numerical algorithms shows that in general the coupling procedures are numerically stable, but care is required to achieve accuracy when using very few timesteps per period of natural oscillation of the structure. The relevance of the analysis to fully three-dimensional applications is discussed.

Subject classifications: AMS(MOS): 65M10,76N10

Key words and phrases: Aeroelasticity, numerical analysis, boundary conditions This work was supported by Rolls-Royce plc, DTI and EPSRC.

Oxford University Computing Laboratory

Numerical Analysis Group

Wolfson Building

Parks Road

Oxford, England OX1 3QD

E-mail: giles@comlab.oxford.ac.uk

April, 1997 


\section{Introduction}

This analysis is motivated by interest in numerical procedures for coupling an unsteady CFD computation to an unsteady structural dynamics model to predict aeroelastic behaviour. Extremely large 3D computations of this sort are necessary to accurately predict the onset of flutter in both turbomachinery and aircraft applications. One approach to the numerical approximation of this problem is the use of a single consistent, fully-coupled discretisation modelling both the structure and the fluid as a continuum whose dynamics is governed by partial differential equations, plus boundary conditions at the interfaces. However, for the solid the relevant p.d.e. is the equation of motion for an elastic solid, while for the fluid the appropriate equations are the Navier-Stokes equations with suitable turbulence modelling. Moreover, each has its own characteristic length scales and time scales. Therefore, the production of a single fully-coupled code for the combined aeroelastic application is at least as much work as writing the individual programs for the separate solid and fluid applications. Since there are existing codes which accurately and efficiently solve these individual problems, the more practical approach is to investigate how best to couple such codes together to analyse aeroelastic problems $[4,5,8,9]$. There is a concern that the coupling procedure may introduce a spurious numerical instability, unrelated to the real flutter instabilities which are the focus of engineering attention. The original aim of this study was to investigate this possibility, but an additional objective which developed during the research was to investigate the accuracy of the resulting coupled analysis.

The general theory for the analysis of numerical interface or boundary condition instabilities is well-established but can be complicated to apply in practice $[3,6,11,13]$. In this paper we simplify the analysis by restricting attention to a simple 1D model problem. The first section of the paper constructs the model and tries to justify its relevance to the real 3D engineering problem. The next section presents stability analyses for two different discretisations of the fluid equations and a number of different treatments of the coupling to the structural equations. This reveals that in general there is no spurious numerical instability, but there may be a problem with the accuracy of the numerical approximation and solid/fluid coupling which may lead to a poor approximation of the real stability properties of the aeroelastic system. The third section presents an alternative discretisation of the structural dynamics and associated aerodynamic boundary conditions which is stable and much more accurate. The final sections give some further discussion of the relevance of the analysis to real 3D engineering applications, and make some conclusions and recommendations for future work. 


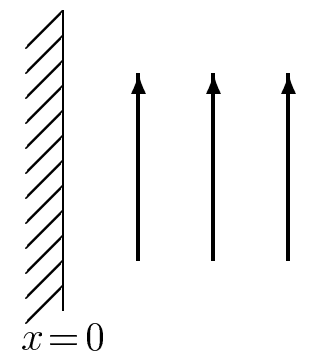

Figure 1: Parallel flow past a flat wall

\section{Analytic equations}

As shown in Fig. 1, we start by considering a steady 2D parallel flow with velocity $(0, V)^{T}$ in the region $x>0$. The equations describing isentropic linearised perturbations to this compressible 2D flow field are

$$
\frac{\partial}{\partial t}\left(\begin{array}{l}
p \\
u \\
v
\end{array}\right)+\left(\begin{array}{ccc}
0 & \rho c^{2} & 0 \\
\frac{1}{\rho} & 0 & 0 \\
0 & 0 & 0
\end{array}\right) \frac{\partial}{\partial x}\left(\begin{array}{c}
p \\
u \\
v
\end{array}\right)+\left(\begin{array}{lll}
V & 0 & \rho c^{2} \\
0 & V & 0 \\
\frac{1}{\rho} & 0 & V
\end{array}\right) \frac{\partial}{\partial y}\left(\begin{array}{c}
p \\
u \\
v
\end{array}\right)=0
$$

where $\rho, c$ are the average density and speed of sound and $p, u, v$ are the unsteady perturbations to the pressure and the two velocity components.

If the wall oscillates so that its position $x_{w}(t)$ is independent of $y$, then the resulting fluid perturbation will also be independent of $y$, and there will be no perturbation to the velocity in the $y$-direction. This reduces the linear unsteady aerodynamic equations to the simple form

$$
\frac{\partial}{\partial t}\left(\begin{array}{l}
p \\
u
\end{array}\right)+\left(\begin{array}{ll}
0 & \rho c^{2} \\
\frac{1}{\rho} & 0
\end{array}\right) \frac{\partial}{\partial x}\left(\begin{array}{l}
p \\
u
\end{array}\right)=0
$$

which is the same set of equations as those describing perturbations to a 1D stationary flow with the same density and speed of sound. It is interesting, and perhaps surprising, that these equations do not depend on the Mach number of the mean flow; this is because the assumption of no variation in the $y$-direction allows a Lagrangian transformation to new coordinates $x^{*}=x, y^{*}=y-V t$, relative to which the mean flow is indeed stationary.

Having justified the simple 1D aerodynamic equations, the other aspect of the model formulation is the interaction between the aerodynamics and the motion of the wall. One boundary condition is the linearised kinematic condition that the flow velocity relative to the moving wall is zero.

$$
\dot{x}_{w}(t)=u(0, t) .
$$

The dynamics of the wall's motion are modelled by a simple mass-spring system subject to the external unsteady aerodynamic pressure.

$$
m \ddot{x}_{w}+m \omega_{0}^{2} x_{w}=-p(0, t) .
$$


Here $m$ represents the mass per unit area and $\omega_{0}$ is the natural frequency of oscillation in the absence of any aerodynamic coupling. This equation will be referred to as the scalar version of the wall dynamic equation. Some numerical discretisations start from an equivalent coupled system of first order o.d.e.'s,

$$
\frac{d w}{d t}+A w=P
$$

where

$$
w=\left(\begin{array}{c}
\omega_{0} x_{w} \\
\dot{x}_{w}
\end{array}\right), \quad A=\left(\begin{array}{cc}
0 & -\omega_{0} \\
\omega_{0} & 0
\end{array}\right), \quad P=\left(\begin{array}{c}
0 \\
-\frac{p(0, t)}{m}
\end{array}\right) .
$$

This will be referred to as the vector version of the equation.

This simple structural model seems far removed from the original representation of a continuum elastic solid discussed in the Introduction. In fact, it is common to represent the dynamics of an oscillating blade in terms of a very limited number of structural modes; these are usually obtained using a finite element approximation of the elastic solid vibrating in the absence of any external aerodynamics. The modes with the lowest natural frequency are the ones which have the greatest potential for flutter and large forced excitation, which is why higher modes are neglected. The lowest mode is usually a simple bending mode whose nature is very similar to the simple undamped mass-spring system. Even a torsional mode can be viewed locally (near the blade's surface) as being similar to the model problem in that there are no large variations in the tangential direction and so $1 \mathrm{D}$ aerodynamics is a good local approximation. In other $2 \mathrm{D}$ and $3 \mathrm{D}$ computations with numerical instabilities at interfaces, it is generally true that any instability will first occur with a purely $1 \mathrm{D}$ eigenmode with a spatial variation in the direction normal to the interface but no variation along the interface. Thus the 1D model problem should be appropriate in trying to identify the possibility of a purely numerical instability.

This simple model problem exhibits aerodynamic damping of the wall's oscillation. To determine this it is helpful to perform a change of variables in the aerodynamic equations. Characteristic variables defined by

$$
\begin{aligned}
& q=p+\rho c u, \\
& r=p-\rho c u,
\end{aligned}
$$

satisfy the uncoupled equations

$$
\begin{aligned}
& \frac{\partial q}{\partial t}+c \frac{\partial q}{\partial x}=0 \\
& \frac{\partial r}{\partial t}-c \frac{\partial r}{\partial x}=0
\end{aligned}
$$

The acoustic wave represented by $q$ travels right with velocity $c$, while the other acoustic wave represented by $r$ travels left with velocity $-c$. We need to impose 
a radiation condition at $x=\infty$ requiring that sufficiently far from the wall all perturbations are travelling away from the wall, not towards it. Thus we require that $r \rightarrow 0$ as $x \rightarrow \infty$.

Using the characteristic variables, the equations for the wall are

$$
\begin{aligned}
\dot{x}_{w} & =\frac{1}{2 \rho c}(q(0, t)-r(0, t)), \\
m \ddot{x}_{w}+m \omega_{0}^{2} x_{w} & =-\frac{1}{2}(q(0, t)+r(0, t)) .
\end{aligned}
$$

We now consider solutions of the form

$$
\begin{aligned}
x_{w}(t) & =X e^{i \omega t} \\
q(x, t) & =Q e^{i \omega\left(t-\frac{x}{c}\right)} \\
r(x, t) & =0
\end{aligned}
$$

with $X$ and $Q$ being complex constants. The real physical variables correspond to the real components of these complex expressions. These solutions satisfy the necessary equations and boundary conditions provided that

$$
\begin{aligned}
i \omega X & =\frac{1}{2 \rho c} Q, \\
\left(-m \omega^{2}+m \omega_{0}^{2}\right) X & =-\frac{1}{2} Q,
\end{aligned}
$$

for which non-trivial solutions exist only if

$$
-m \omega^{2}+i \omega \rho c+m \omega_{0}^{2}=0 \quad \Longrightarrow \quad \frac{\omega}{\omega_{0}}=i d \pm \sqrt{1-d^{2}}
$$

where $d=\frac{\rho c}{2 m \omega_{0}}$ is the non-dimensional damping factor. In real turbomachinery applications modelled using a structure with a single degree of freedom, the level of damping is very small, usually in the range 0.005-0.02. For aircraft applications concerned with wing aeroelasticity values in the range 0.05-0.2 are more typical. Assuming that $d$ is small,

$$
\frac{\omega}{\omega_{0}}=i d \pm\left(1-\frac{1}{2} d^{2}\right)+O\left(d^{4}\right) .
$$

Taking the positive root without any loss of generality (since the real variables correspond to the real component only) gives

$$
e^{i \omega t} \approx e^{i \omega_{0} t-d \omega_{0} t}
$$

and so the fractional reduction in the wall's oscillation amplitude in one period of oscillation is

$$
1-e^{-2 \pi d} \approx 2 \pi d .
$$


The model equations do not have any terms describing energy dissipation. It can be shown that this reduction in the vibrational energy of the wall is in fact exactly equal to the acoustic energy radiated during the period of oscillation.

In considering discretisations of the model equations, the central question is whether the discrete approximation allows unstable exponentially growing solutions with a timescale which is much smaller than the period of oscillation $\frac{2 \pi}{\omega_{0}}$. If there is no numerical instability, then the secondary question is how accurately the aerodynamic damping is modelled by the discretisation. 


\section{Structural algorithms based only on wall displacement}

In this section we consider coupled aeroelastic discretisations in which the scalar form of the wall dynamic equation is approximated using central time differencing.

$$
\frac{m}{\Delta t^{2}}\left(x_{w}^{(n+1)}-2 x_{w}^{(n)}+x_{w}^{(n-1)}\right)+m \omega_{0}^{2} x_{w}^{(n)}=-\frac{1}{2}\left(q_{0}^{(n)}+r_{0}^{(n)}\right)
$$

A number of different discretisations of the kinematic condition will be analysed.

\subsection{Upwind aerodynamic discretisation}

The first algorithms are based on upwind discretisation of the aerodynamic equations. Using forward time differencing and upwind spatial differencing, the interior equations are

$$
\begin{array}{ll}
q_{j}^{(n+1)}=q_{j}^{(n)}-\frac{c \Delta t}{\Delta x}\left(q_{j}^{(n)}-q_{j-1}^{(n)}\right), & j=1,2,3, \ldots \\
r_{j}^{(n+1)}=r_{j}^{(n)}+\frac{c \Delta t}{\Delta x}\left(r_{j+1}^{(n)}-r_{j}^{(n)}\right), & j=0,1,2, \ldots
\end{array}
$$

The stability analysis considers the possible existence of a G-R (GodunovRyabenkii) normal mode $[3,11]$ of the form

$$
\begin{aligned}
& x_{w}^{(n)}=X z^{n} \\
& q_{j}^{(n)}=Q z^{n} \kappa_{q}^{j} \\
& r_{j}^{(n)}=R z^{n} \kappa_{r}^{j},
\end{aligned}
$$

with $|z|>1$ corresponding to an unstable mode. $\kappa_{q}$ and $\kappa_{r}$ are necessarily related to $z$ through the interior equations which require that

$$
\begin{aligned}
& z=1-\lambda\left(1-\kappa_{q}^{-1}\right) \\
& z=1+\lambda\left(\kappa_{r}-1\right) .
\end{aligned}
$$

$\lambda$ is the CFL parameter $\frac{c \Delta t}{\Delta x}$ and must satisfy the Fourier stability restriction $\lambda \leq 1$. It can be shown that if $|z|>1$, then $\left|\kappa_{q}\right|<1$ and $\left|\kappa_{r}\right|>1$. Hence, to satisfy the discrete equivalent of the radiation condition that all variables tend to zero as $j \rightarrow \infty$, it is necessary that $R=0$.

\subsubsection{First order coupling}

The final discrete equation is the kinematic compatibility condition. A simple first order approximation of this is

$$
\frac{1}{\Delta t}\left(x_{w}^{(n+1)}-x_{w}^{(n)}\right)=\frac{1}{2 \rho c}\left(q_{0}^{(n+1)}-r_{0}^{(n+1)}\right) .
$$


Inserting the assumed G-R mode into the wall dynamic equation and this kinematic equation yields the following two equations,

$$
\begin{aligned}
\left(\frac{m}{\Delta t^{2}}\left(z-2+z^{-1}\right)+m \omega_{0}^{2}\right) X & =-\frac{1}{2} Q \\
\frac{z-1}{\Delta t} X & =\frac{z}{2 \rho c} Q
\end{aligned}
$$

for which there are non-trivial solutions only if

$$
z-2+z^{-1}+\left(\omega_{0} \Delta t\right)^{2}=-\frac{\rho c \Delta t}{m}\left(1-z^{-1}\right)=-2 d \omega_{0} \Delta t\left(1-z^{-1}\right),
$$

where $d$ is still the non-dimensional damping factor $d=\frac{\rho c}{2 m \omega_{0}}$. Multiplying by $z$ produces a quadratic equation. When $d=0$ the roots of this are

$$
\begin{aligned}
z & =1-\frac{1}{2}\left(\omega_{0} \Delta t\right)^{2} \pm \sqrt{\left(1-\frac{1}{2}\left(\omega_{0} \Delta t\right)^{2}\right)^{2}-1} \\
& =1-\frac{1}{2}\left(\omega_{0} \Delta t\right)^{2} \pm i \omega_{0} \Delta t \sqrt{1-\frac{1}{4}\left(\omega_{0} \Delta t\right)^{2}}
\end{aligned}
$$

If $\omega_{0} \Delta t \leq 2$ then the two roots are a complex conjugate pair of unit magnitude, while if $\omega_{0} \Delta t>2$ then the two roots are real and negative, with one having a magnitude greater than unity, giving unstable exponential growth. Thus, numerical stability in the absence of any aerodynamic coupling requires $\Delta t \leq \frac{2}{\omega_{0}}$. This means that there must be more than 3 timesteps per period of oscillation, but it is clear that many more timesteps than this are required for accuracy and so this stability criterion is not significant.

When $0<d \ll 1$, we look for roots of the quadratic for which $|z|=1$, corresponding to the threshold of instability. $z=1$ is never a solution for $\Delta t>0$. $z=-1$ is a solution when

$$
4-\left(\omega_{0} \Delta t\right)^{2}-2 d\left(\omega_{0} \Delta t\right)=0 \quad \Longrightarrow \quad \omega_{0} \Delta t \approx 2
$$

The only other roots with $|z|=1$ must occur as a complex conjugate pair, but in that case their magnitude must be $\sqrt{1-2 d \omega_{0} \Delta t}$ which is less than 1 for $\omega_{0} \Delta t<2$. Thus $\omega_{0} \Delta t<2$ remains the approximate stability limit of this coupled problem. Assuming there are sufficient timesteps per period to achieve an accurate solution, there is therefore no numerical stability problem.

To determine the accuracy let $z=e^{i \omega \Delta t}$ which gives

$$
e^{i \omega \Delta t}-2+e^{-i \omega \Delta t}+\left(\omega_{0} \Delta t\right)^{2}=-2 d \omega_{0} \Delta t\left(1-e^{-i \omega \Delta t}\right) .
$$

For $0<d \ll 1$ it is convenient to consider $\omega$ as a function of $d$ as well as $\Delta t$ and $\omega_{0}$, and perform a Taylor series expansion in $d$ to obtain

$$
\omega=\left.\omega\right|_{d=0}+\left.d \frac{\partial \omega}{\partial d}\right|_{d=0}+O\left(d^{2}\right) .
$$


When $d=0$, Equation (3.10) gives

$$
e^{i \omega \Delta t}-2+e^{-i \omega \Delta t}+\left(\omega_{0} \Delta t\right)^{2}=0 \quad \Longrightarrow \quad 4 \sin ^{2}\left(\frac{\omega \Delta t}{2}\right)=\left(\omega_{0} \Delta t\right)^{2} .
$$

Differentiating Equation (3.10) with respect to $d$ then gives

$$
i \Delta t\left(e^{i \omega \Delta t}-e^{-i \omega \Delta t}\right) \frac{\partial \omega}{\partial d}=-2 \omega_{0} \Delta t\left(1-e^{-i \omega \Delta t}\right) \quad \Longrightarrow \quad \frac{\partial \omega}{\partial d}=\omega_{0}\left(i+\tan \left(\frac{\omega \Delta t}{2}\right)\right)
$$

If it is also assumed that $\omega_{0} \Delta t \ll 1$, then

$$
\sin \left(\frac{\omega \Delta t}{2}\right) \approx \frac{\omega \Delta t}{2}-\frac{1}{6}\left(\frac{\omega \Delta t}{2}\right)^{3}
$$

and hence

$$
\frac{\omega}{\omega_{0}} \approx 1+\frac{1}{24}\left(\omega_{0} \Delta t\right)^{2}+d\left(i+\frac{1}{2} \omega_{0} \Delta t\right)
$$

The first order error in the coupling produces a first-order shift in the real part of the frequency, but no error in the important imaginary part which gives the predicted damping. However, in a real aeroelastic computation in which the aerodynamics causes a frequency shift as well as a damping effect, the first-order coupling error would probably also produce a first-order error in the predicted damping. For a typical flutter frequency and a timestep limited by the CFL stability restriction $\frac{c \Delta t}{\Delta x}<1$ for a typical grid resolution, $\omega_{0} \Delta t$ will be in the range $10^{-3}-10^{-2}$. In this case, the errors in both the frequency and the damping are negligible compared to other errors such as modelling approximations and uncertainty about structural damping factors.

This conclusion about the adequacy of first order coupling changes entirely when one considers implicit methods. Replacing the forward time differencing of the aerodynamics by backward time differencing gives the following algorithm for the aerodynamics.

$$
\begin{array}{ll}
q_{j}^{(n+1)}=q_{j}^{(n)}-\frac{c \Delta t}{\Delta x}\left(q_{j}^{(n+1)}-q_{j-1}^{(n+1)}\right), & j=1,2,3, \ldots \\
r_{j}^{(n+1)}=r_{j}^{(n)}+\frac{c \Delta t}{\Delta x}\left(r_{j+1}^{(n+1)}-r_{j}^{(n+1)}\right), & j=0,1,2, \ldots
\end{array}
$$

All of the previous analysis remains valid. This surprising fact is because the wall coupling equations do not depend on the interior equations once it is determined that $r_{j}^{(n)}$ is zero throughout the domain in order to satisfy the discrete radiation condition. The conclusions about the accuracy change because the timestep is no longer limited by the CFL condition. Instead, $\omega_{0} \Delta t$ will typically be $O\left(10^{-1}\right)$. It is the computational efficiency of this much larger timestep which is the attraction of using implicit methods for flutter analysis and other unsteady flow calculations at low reduced frequencies $[7,10]$ However, as a consequence the first order coupling is no longer sufficiently accurate. 


\subsubsection{An explicit second order coupling}

A second-order accurate coupling is achieved by changing the kinematic discretisation to

$$
\frac{1}{\Delta t}\left(\frac{3}{2} x_{w}^{(n+1)}-2 x_{w}^{(n)}+\frac{1}{2} x_{w}^{(n-1)}\right)=\frac{1}{2 \rho c}\left(q_{0}^{(n+1)}-r_{0}^{(n+1)}\right)
$$

which leads to the following modified equation for $\mathrm{z}$.

$$
z-2+z^{-1}+\left(\omega_{0} \Delta t\right)^{2}=-2 d \omega_{0} \Delta t\left(\frac{3}{2}-2 z^{-1}+\frac{1}{2} z^{-2}\right)
$$

Multiplying by $z^{2}$ now gives a cubic equation in $z$. In the limit as $d \rightarrow 0$ two of the roots are the same as before and the third is $z=0$. This third root is only slightly perturbed when $d>0$ and so remains strongly stable. To find the perturbation to the other two roots it is convenient again to make the substitution $z=e^{i \omega \Delta t}$ giving

$$
e^{i \omega \Delta t}-2+e^{-i \omega \Delta t}+\left(\omega_{0} \Delta t\right)^{2}=-2 d \omega_{0} \Delta t\left(\frac{3}{2}-2 e^{-i \omega \Delta t}+\frac{1}{2} e^{-2 i \omega \Delta t}\right) .
$$

Differentiating with respect to $d$ gives

$$
\begin{aligned}
i \Delta t\left(e^{i \omega \Delta t}-e^{-i \omega \Delta t}\right) \frac{\partial \omega}{\partial d} & =-2 \omega_{0} \Delta t\left(\frac{3}{2}-2 e^{-i \omega \Delta t}+\frac{1}{2} e^{-2 i \omega \Delta t}\right) \\
\Longrightarrow \frac{\partial \omega}{\partial d} & =\omega_{0}\left(i+2 i \sin ^{2}\left(\frac{\omega \Delta t}{2}\right)+2 \tan \left(\frac{\omega \Delta t}{2}\right) \sin ^{2}\left(\frac{\omega \Delta t}{2}\right)\right)(3 .
\end{aligned}
$$

The two roots which are neutrally stable when $d=0$ and $\omega_{0} \Delta t<2$ have corresponding real values for $\omega$. The imaginary part of $\frac{\partial \omega}{\partial d}$ is then positive showing that the perturbed roots are stable. The stability boundary therefore remains $\omega_{0} \Delta t<2$.

If $\omega_{0} \Delta t \ll 1$ then

$$
\frac{\omega}{\omega_{0}} \approx 1+i d+\frac{1}{24}\left(\omega_{0} \Delta t\right)^{2}+\frac{1}{2} i d\left(\omega_{0} \Delta t\right)^{2} .
$$

Because of the improved accuracy of the kinematic discretisation the error is now second order in $\omega_{0} \Delta t$. If $\omega_{0} \Delta t=0.1$, corresponding to approximately 60 timesteps per period, then the error is probably acceptable; if $\omega_{0} \Delta t=0.3$, corresponding to approximately 20 timesteps per period, then the error is probably unacceptable.

\subsubsection{An unstable second order coupling}

Another second-order accurate discretisation of the kinematic condition is

$$
\frac{1}{\Delta t}\left(x_{w}^{(n+1)}-x_{w}^{(n)}\right)=\frac{1}{4 \rho c}\left(q_{0}^{(n)}+q_{0}^{(n+1)}-r_{0}^{(n)}-r_{0}^{(n+1)}\right)
$$


which leads to the following modified equation for $\mathrm{z}$.

$$
z-2+z^{-1}+\left(\omega_{0} \Delta t\right)^{2}=-4 \omega_{0} \Delta t \frac{z-1}{z+1}
$$

Multiplying by $z+1$ gives a cubic equation in $z$. In the limit as $d \rightarrow 0$ the spurious root is $z=-1$. Differentiating the cubic equation with respect to $d$ for $z=-1$ gives

$$
\frac{\partial z}{\partial d}=-\frac{8 \omega_{0} \Delta t}{4-\left(\omega_{0} \Delta t\right)^{2}}
$$

and so

$$
z=-1-\frac{8 d \omega_{0} \Delta t}{4-\left(\omega_{0} \Delta t\right)^{2}}+O\left(d^{2}\right)
$$

This root has magnitude greater than 1 for $\omega_{0} \Delta t<2$ and the other two roots are unstable for larger values of $\Delta t$. Therefore, the algorithm is unconditionally unstable.

\subsubsection{An implicit second order coupling}

Yet another second-order accurate discretisation is

$$
\frac{1}{2 \Delta t}\left(x_{w}^{(n+2)}-x_{w}^{(n)}\right)=\frac{1}{2 \rho c}\left(q_{0}^{(n+1)}-r_{0}^{(n+1)}\right)
$$

which leads to the following modified equation for $\mathrm{z}$.

$$
z-2+z^{-1}+\left(\omega_{0} \Delta t\right)^{2}=-d \omega_{0} \Delta t\left(z-z^{-1}\right)
$$

Multiplying by $z$ gives a quadratic equation with no spurious roots. Substituting $z=e^{i \omega \Delta t}$ and differentiating yields

$$
\frac{\partial \omega}{\partial d}=i \omega_{0}
$$

and so the perturbed roots are stable for $\omega_{0} \Delta t<2$. If $\omega_{0} \Delta t \ll 1$ then

$$
\frac{\omega}{\omega_{0}} \approx 1+\frac{1}{24}\left(\omega_{0} \Delta t\right)^{2}+i d
$$

It is interesting that the $O(d)$ damping term is obtained exactly, independent of the value of $\omega_{0} \Delta t$ as long as it satisfies the stability constraint. It is not clear whether this property would still hold for real 3D applications.

The problem with this kinematic discretisation is that it is now an implicit algorithm since the surface pressure $p_{0}^{(n)}$ depends on $x_{w}^{(n+1)}$, and vice versa. This implicitness is awkward because in a 3D application it means that the aerodynamic variables at all grid points on the surface of the vibrating blade are coupled 
through the structural boundary conditions. The difficulty can be overcome by a predictor/corrector implementation:

$$
\begin{gathered}
p_{0}^{*}=2 p_{0}^{(n-1)}-p_{0}^{(n-2)} \\
\left.\begin{array}{c}
\frac{m}{\Delta t^{2}}\left(x_{w}^{*}-2 x_{w}^{(n)}+x_{w}^{(n-1)}\right)+m \omega_{0}^{2} x_{w}^{(n)}=-p_{0}^{*} \\
p_{0}^{(n)}=p_{0}^{(n)}\left(x_{w}^{(n-1)}, x_{w}^{(n)}, x_{w}^{*}, q_{j}^{(n-1)}, r_{j}^{(n-1)}\right) \\
\frac{m}{\Delta t^{2}}\left(x_{w}^{(n+1)}-2 x_{w}^{(n)}+x_{w}^{(n-1)}\right)+m \omega_{0}^{2} x_{w}^{(n)}=-p_{0}^{(n)}
\end{array}\right\} \text { Predictor } \\
\text { Corrector }
\end{gathered}
$$

In the prediction stage, a first approximation for the surface pressure $p_{0}^{(n)}$ is given by linear extrapolation from the previous two timesteps, and this is used to obtain a first estimate for $x_{w}^{(n+1)}$. In the correction stage, the predicted value $x_{w}^{*}$ is used in conjunction with the discrete aerodynamic equations and kinematic boundary condition to calculate a corrected value for $p_{0}^{(n)}$; this is then used to calculate a corrected value for $x_{w}^{(n+1)}$. The error introduced by this predictor/corrector approximation to the original implicit algorithm is $O\left(d^{2}\right)$ which is negligible if $d \ll 1$. 


\subsection{Lax-Wendroff discretisation}

The Lax-Wendroff discretisation of the pressure equation at grid nodes other than the wall node is

$$
\begin{aligned}
p_{j}^{(n+1)}=p_{j}^{(n)}-\frac{\rho c^{2} \Delta t}{2 \Delta x} & \left(u_{j+1}^{(n)}-u_{j-1}^{(n)}\right)+\frac{c^{2} \Delta t^{2}}{2 \Delta x^{2}}\left(p_{j+1}^{(n)}-2 p_{j}^{(n)}+p_{j-1}^{(n)}\right) \\
=p_{j}^{(n)}-\frac{\rho c^{2} \Delta t}{\Delta x} & \left\{\left(\frac{1}{2}\left(u_{j+1}^{(n)}+u_{j}^{(n)}\right)-\frac{\Delta t}{2 \rho \Delta x}\left(p_{j+1}^{(n)}-p_{j}^{(n)}\right)\right)\right. \\
& \left.-\left(\frac{1}{2}\left(u_{j}^{(n)}+u_{j-1}^{(n)}\right)-\frac{\Delta t}{2 \rho \Delta x}\left(p_{j}^{(n)}-p_{j-1}^{(n)}\right)\right)\right\}
\end{aligned}
$$

where

$$
\frac{1}{2}\left(u_{j+1}^{(n)}+u_{j}^{(n)}\right)-\frac{\Delta t}{2 \rho \Delta x}\left(p_{j+1}^{(n)}-p_{j}^{(n)}\right)
$$

represents a second order approximation to $u(x, t)$ at $x=\left(j+\frac{1}{2}\right) \Delta x, t=\left(n+\frac{1}{2}\right) \Delta t$.

The corresponding discrete equation for the velocity is

$$
u_{j}^{(n+1)}=u_{j}^{(n)}-\frac{\Delta t}{2 \rho \Delta x}\left(p_{j+1}^{(n)}-p_{j-1}^{(n)}\right)+\frac{c^{2} \Delta t^{2}}{2 \Delta x^{2}}\left(u_{j+1}^{(n)}-2 u_{j}^{(n)}+u_{j-1}^{(n)}\right) .
$$

Combining these two equations gives the Lax-Wendroff equations for the characteristic variables.

$$
\begin{aligned}
q_{j}^{(n+1)} & =q_{j}^{(n)}-\frac{c \Delta t}{2 \Delta x}\left(q_{j+1}^{(n)}-q_{j-1}^{(n)}\right)+\frac{c^{2} \Delta t^{2}}{2 \Delta x^{2}}\left(q_{j+1}^{(n)}-2 q_{j}^{(n)}+q_{j-1}^{(n)}\right), \\
r_{j}^{(n+1)} & =r_{j}^{(n)}-\frac{c \Delta t}{2 \Delta x}\left(r_{j+1}^{(n)}-r_{j-1}^{(n)}\right)+\frac{c^{2} \Delta t^{2}}{2 \Delta x^{2}}\left(r_{j+1}^{(n)}-2 r_{j}^{(n)}+r_{j-1}^{(n)}\right) .
\end{aligned}
$$

At the wall, the computational cell is half the usual size, extending from $x=0$ to $x=\frac{1}{2} \Delta x$. In addition, the velocity of the wall at $t=\left(n+\frac{1}{2}\right) \Delta t$ is well approximated by $\Delta t^{-1}\left(x_{w}^{(n+1)}-x_{w}^{(n)}\right)$. Thus the discrete pressure equation for $j=0$ becomes

$$
p_{0}^{(n+1)}=p_{0}^{(n)}-\frac{2 \rho c^{2} \Delta t}{\Delta x}\left\{\left(\frac{1}{2}\left(u_{1}^{(n)}+u_{0}^{(n)}\right)-\frac{\Delta t}{2 \rho \Delta x}\left(p_{1}^{(n)}-p_{0}^{(n)}\right)\right)-\frac{1}{\Delta t}\left(x_{w}^{(n+1)}-x_{w}^{(n)}\right)\right\}
$$

Using the same first order coupling as for the upwind discretisation, the discrete velocity equation at the wall is repaced by the kinematic condition

$$
\frac{1}{\Delta t}\left(x_{w}^{(n+1)}-x_{w}^{(n)}\right)=u_{0}^{(n+1)},
$$

and the dynamic equation is again

$$
\frac{m}{\Delta t^{2}}\left(x_{w}^{(n+1)}-2 x_{w}^{(n)}+x_{w}^{(n-1)}\right)+m \omega_{0}^{2} x_{w}^{(n)}=-p_{0}^{(n)} .
$$


We again consider the possibility of a G-R normal mode of the form

$$
\begin{aligned}
x_{w}^{(n)} & =X z^{n} \\
q_{j}^{(n)} & =Q z^{n} \kappa_{q}^{j} \\
r_{j}^{(n)} & =R z^{n} \kappa_{r}^{j} .
\end{aligned}
$$

$\kappa_{q}$ and $\kappa_{r}$ are related to $z$ through the interior equations which require that

$$
\begin{aligned}
& z=1-\frac{1}{2} \lambda\left(\kappa_{q}-\kappa_{q}^{-1}\right)+\frac{1}{2} \lambda^{2}\left(\kappa_{q}-2+\kappa_{q}^{-1}\right) \\
& z=1+\frac{1}{2} \lambda\left(\kappa_{r}-\kappa_{r}^{-1}\right)+\frac{1}{2} \lambda^{2}\left(\kappa_{r}-2+\kappa_{r}^{-1}\right)
\end{aligned}
$$

where $\lambda \equiv \frac{c \Delta t}{\Delta x}$ and $\lambda \leq 1$ as before. Solving the quadratic equation for $\kappa_{q}$ gives

$$
\kappa_{q}=\frac{z-1+\lambda^{2} \pm \sqrt{(z-1)^{2}+(2 z-1) \lambda^{2}}}{-\lambda+\lambda^{2}}
$$

It can be shown that for $|z|>1$ one of these two roots has magnitude greater than unity while the other has magnitude less than unity. To satisfy the requirement that the discrete solution must approach zero as $j \rightarrow \infty$ we must choose the latter root, and so

$$
\kappa_{q}=\frac{z-1+\lambda^{2}-\sqrt{(z-1)^{2}+(2 z-1) \lambda^{2}}}{-\lambda+\lambda^{2}}
$$

with the complex square root being determined suitably when its argument is complex. Similarly, solving the quadratic equation for $\kappa_{r}$, the root with magnitude less than unity is

$$
\kappa_{r}=\frac{z-1+\lambda^{2}-\sqrt{(z-1)^{2}+(2 z-1) \lambda^{2}}}{\lambda+\lambda^{2}}
$$

with the complex square root being determined in the same manner as in the definition of $\kappa_{q}$.

Three equations are now needed to determine the relationship between the complex constants $X, Q, R$. The first two are the kinematic boundary condition which requires that

$$
Q-R=\frac{2 \rho c}{\Delta t}\left(1-z^{-1}\right) X
$$

and the wall dynamic equation which requires that

$$
\left(\frac{m}{\Delta t^{2}}\left(z-2+z^{-1}\right)+m \omega_{0}^{2}\right) X=-\frac{1}{2}(Q+R) .
$$

The third equation comes from substituting the assumed G-R mode into the pressure equation for $j=0$, which gives

$$
\left(z-1+\lambda-\left(-\lambda+\lambda^{2}\right) \kappa_{q}\right) Q+\left(z-1-\lambda-\left(\lambda+\lambda^{2}\right) \kappa_{r}\right) R=\frac{4 \rho c}{\Delta t} \lambda(z-1) X .
$$


Substituting for $\kappa_{q}$ and $\kappa_{r}$ gives

$$
\sqrt{(z-1)^{2}+(2 z-1) \lambda^{2}}(Q+R)+\lambda(Q-R)=\frac{4 \rho c}{\Delta t} \lambda(z-1) X .
$$

Combining this with Equation (3.42) gives

$$
\sqrt{(z-1)^{2}+(2 z-1) \lambda^{2}}(Q+R)=\frac{2 \rho c \lambda}{\Delta t}\left(2 z-3+z^{-1}\right) X
$$

and hence there are no non-trivial solutions unless

$$
z-2+z^{-1}+\left(\omega_{0} \Delta t\right)^{2}=-2 d \omega_{0} \Delta t \frac{\lambda\left(2 z-3+z^{-1}\right)}{\sqrt{(z-1)^{2}+(2 z-1) \lambda^{2}}} .
$$

Letting $z=\exp (i \omega \Delta t)$ and using the standard asymptotic analysis assuming $d \ll 1$ gives

$$
\frac{\partial \omega}{\partial d}=\omega_{0} f(\omega \Delta t, \lambda)
$$

where the function $f(\omega \Delta t, \lambda)$ is defined as

$$
\begin{aligned}
f(\omega \Delta t, \lambda) & \equiv \frac{2 i \lambda\left(2 e^{i \omega \Delta t}-3+e^{-i \omega \Delta t}\right)}{\left(e^{i \omega \Delta t}-e^{-i \omega \Delta t}\right) \sqrt{\left(e^{i \omega \Delta t}-1\right)^{2}+\left(2 e^{i \omega \Delta t}-1\right) \lambda^{2}}} \\
& =\frac{i\left(1+3 i \tan \left(\frac{1}{2} \omega \Delta t\right)\right)}{\sqrt{\left(e^{i \omega \Delta t}-1\right)^{2} \lambda^{-2}+2 e^{i \omega \Delta t}-1}}
\end{aligned}
$$

If it is assumed that the acoustic waves are well resolved on the computational grid then

$$
\Delta x \ll \frac{c}{\omega} \quad \Longrightarrow \quad \omega \Delta t \ll \lambda .
$$

Hence,

$$
\sqrt{\left(e^{i \omega \Delta t}-1\right)^{2} \lambda^{-2}+2 e^{i \omega \Delta t}-1} \approx 1+i \omega \Delta t \quad \Longrightarrow \quad f(\omega \Delta t, \lambda) \approx i+\frac{1}{2} \omega \Delta t
$$

and so the perturbed roots are stable and first order accurate as with the upwind discretisation of the aerodynamics. For larger values of $\omega \Delta t$ it would be necessary to resort to direct numerical evaluation of $f(\omega \Delta t, \lambda)$ to investigate the stability and accuracy of the scheme. 


\section{Structural algorithms based on wall displacement and velocity}

In this section we consider coupled aeroelastic discretisations based on the vector form of the wall dynamic equation. An advantage of this approach is that by calculating both the displacement and velocity at each timestep the kinematic boundary condition becomes simply

$$
\dot{x}_{w}^{(n)}=u_{0}^{(n)} .
$$

\subsection{Trapezoidal integration}

The simplest second order accurate discretisation of the vector form of the wall dynamic equation is trapezoidal integration (also known as the Crank-Nicolson or box method),

$$
\frac{1}{\Delta t}\left(w^{(n+1)}-w^{(n)}\right)+\frac{1}{2} A\left(w^{(n+1)}+w^{(n)}\right)=\frac{1}{2}\left(P^{(n+1)}+P^{(n)}\right),
$$

which can be re-arranged to give

$$
w^{(n+1)}=\left(I+\frac{1}{2} \Delta t A\right)^{-1}\left(\left(I-\frac{1}{2} \Delta t A\right) w^{(n)}+\frac{1}{2} \Delta t\left(P^{(n+1)}+P^{(n)}\right)\right) .
$$

As with the implicit method of the last section, there is the problem that $w^{(n+1)}$ depends on $P^{(n+1)}$, and vice versa; this is again solved using a predictor/corrector procedure.

$$
\left.\begin{array}{rl}
P^{*} & =2 P^{(n)}-P^{(n-1)} \\
w^{*} & =\left(I+\frac{1}{2} \Delta t A\right)^{-1}\left(\left(I-\frac{1}{2} \Delta t A\right) w^{(n)}+\frac{1}{2} \Delta t\left(P^{*}+P^{(n)}\right)\right) \\
P^{(n+1)} & =P^{(n+1)}\left(w^{(n)}, w^{*}, q_{j}^{(n)}, r_{j}^{(n)}\right) \\
w^{(n+1)} & =\left(I+\frac{1}{2} \Delta t A\right)^{-1}\left(\left(I-\frac{1}{2} \Delta t A\right) w^{(n)}+\frac{1}{2} \Delta t\left(P^{(n+1)}+P^{(n)}\right)\right)
\end{array}\right\} \text { Corrector }
$$

As before, the predictor/corrector combination gives results which are within $O\left(d^{2}\right)$ of those which would be obtained from the original implicit coupling. For simplicity, it is the implicit coupling which is now analysed for stability and accuracy.

Assuming that upwind differencing is used for the aerodynamic equations then $r_{j}^{(n)}=0$ for all $j$ and $n$, and hence

$$
P^{(n)}=\left(\begin{array}{c}
0 \\
-\frac{\rho c}{m} \dot{x}_{w}^{(n)}
\end{array}\right)=\left(\begin{array}{c}
0 \\
-2 d \omega_{0} \dot{x}_{w}^{(n)}
\end{array}\right) .
$$


Therefore, Equation (4.2) becomes

$$
\left(\begin{array}{cc}
1 & -\frac{1}{2} \omega_{0} \Delta t \\
\frac{1}{2} \omega_{0} \Delta t & 1+d \omega_{0} \Delta t
\end{array}\right) w^{(n+1)}=\left(\begin{array}{cc}
1 & \frac{1}{2} \omega_{0} \Delta t \\
-\frac{1}{2} \omega_{0} \Delta t & 1-d \omega_{0} \Delta t
\end{array}\right) w^{(n)}
$$

$w^{(n)}=z^{n} W$ is a solution for some non-trivial constant vector $W$ if and only if

$$
\begin{gathered}
\operatorname{det}\left(\begin{array}{cc}
z-1 & -\frac{1}{2} \omega_{0} \Delta t(z+1) \\
\frac{1}{2} \omega_{0} \Delta t(z+1) & z-1+d \omega_{0} \Delta t(z+1)
\end{array}\right)=0, \\
\Longrightarrow z^{2}\left(1+\left(\frac{\omega_{0} \Delta t}{2}\right)^{2}+d \omega_{0} \Delta t\right)-2 z\left(1-\left(\frac{\omega_{0} \Delta t}{2}\right)^{2}\right)+\left(1+\left(\frac{\omega_{0} \Delta t}{2}\right)^{2}-d \omega_{0} \Delta t\right)=0, \\
\Longrightarrow \quad z=\frac{1-\left(\frac{\omega_{0} \Delta t}{2}\right)^{2} \pm \omega_{0} \Delta t \sqrt{1-d^{2}}}{1+\left(\frac{\omega_{0} \Delta t}{2}\right)^{2}+d \omega_{0} \Delta t} .
\end{gathered}
$$

When $d=0,|z|=1$. Setting $z=\exp ( \pm i \omega \Delta t)$, Equation (4.7) can be re-written as

$$
\begin{gathered}
e^{i \omega \Delta t}\left(1+\left(\frac{\omega_{0} \Delta t}{2}\right)^{2}+d \omega_{0} \Delta t\right)-2\left(1-\left(\frac{\omega_{0} \Delta t}{2}\right)^{2}\right)+e^{-i \omega \Delta t}\left(1+\left(\frac{\omega_{0} \Delta t}{2}\right)^{2}-d \omega_{0} \Delta t\right)=0 \\
\Longrightarrow \quad-4 \sin ^{2}\left(\frac{\omega \Delta t}{2}\right)+\left(\frac{\omega_{0} \Delta t}{2}\right)^{2} \cos ^{2}\left(\frac{\omega \Delta t}{2}\right)=0 \\
\Longrightarrow \quad \tan \left(\frac{\omega \Delta t}{2}\right)= \pm \frac{\omega_{0} \Delta t}{2}
\end{gathered}
$$

When $\omega_{0} \Delta t \ll 1$, asymptotic expansion gives

$$
\frac{\omega}{\omega_{0}} \approx 1-\frac{1}{12}\left(\omega_{0} \Delta t\right)^{2}
$$

When $0<d \ll 1$, following the same procedure as in the last section gives

$$
\frac{\partial \omega}{\partial d}=\frac{i \omega_{0}}{1+\left(\frac{\omega_{0} \Delta t}{2}\right)^{2}}
$$

and so for $\omega_{0} \Delta t \ll 1$,

$$
\frac{\omega}{\omega_{0}} \approx 1+i d-\frac{1}{12}\left(\omega_{0} \Delta t\right)^{2}-\frac{1}{4} i d\left(\omega_{0} \Delta t\right)^{2}
$$

Provided $\omega_{0} \Delta t<0.3$, corresponding to there being at least 20 timesteps per period, the real part of the frequency is correct to within $1 \%$ and the damping is correct to within $2 \%$; this is perfectly acceptable accuracy for engineering purposes. 


\subsection{Second order backward differentiation}

Another second order accurate approximation of the dynamic equation is

$$
\frac{1}{\Delta t}\left(\frac{3}{2} w^{(n+1)}-2 w^{(n)}+\frac{1}{2} w^{(n-1)}\right)+A w^{(n+1)}=P^{(n+1)}
$$

This is the method used by Alonso et al [1] for aeroelastic computations in which the fluid is water and so the corresponding value for $d$ is much larger than for aeronautical applications. As with the last method, it can be implemented using a predictor/corrector procedure to avoid the complications of an implicit algorithm. Alonso uses several correction stages within a time-accurate multigrid procedure because of the much larger effect of the fluid dynamics on the structural behaviour [1].

Repeating the analysis, the determinant condition for this discretisation is

$$
\begin{gathered}
\operatorname{det}\left(\begin{array}{cc}
\frac{3}{2} z-2-\frac{1}{2} z^{-1} & -z \omega_{0} \Delta t \\
z \omega_{0} \Delta t & \frac{3}{2} z-2-\frac{1}{2} z^{-1}+2 z d \omega_{0} \Delta t
\end{array}\right)=0, \\
\Longrightarrow \quad\left(\frac{3}{2}-2 z^{-1}+\frac{1}{2} z^{-2}\right)^{2}+2 d \omega_{0} \Delta t\left(\frac{3}{2}-2 z^{-1}+\frac{1}{2} z^{-2}\right)+\left(\omega_{0} \Delta t\right)^{2}=0
\end{gathered}
$$

When $d=0$, this reduces to

$$
\frac{3}{2}-2 z^{-1}+\frac{1}{2} z^{-2}=i \omega_{0} \Delta t .
$$

It can be shown that both roots have less than unit magnitude for all positive values of $\omega_{0} \Delta t$. In particular, when $\omega_{0} \Delta t \ll 1$, one root is a strongly stable spurious root $\left(z \approx \frac{1}{3}\right)$; the other root can be expressed as $z=\exp (i \omega \Delta t)$ for which asymptotic analysis gives

$$
\frac{\omega}{\omega_{0}} \approx 1-\frac{1}{3}\left(\omega_{0} \Delta t\right)^{2}+\frac{1}{4} i\left(\omega_{0} \Delta t\right)^{3}
$$

Differentiating Equation (4.14) gives

$$
\frac{\partial \omega}{\partial d}=\frac{i \omega_{0}}{2 e^{-i \omega \Delta t}-e^{-2 i \omega \Delta t}}
$$

and hence for $\omega_{0} \Delta t \ll 1$,

$$
\frac{\omega}{\omega_{0}} \approx 1-\frac{1}{3}\left(\omega_{0} \Delta t\right)^{2}+\frac{1}{4} i\left(\omega_{0} \Delta t\right)^{3}+i d-i d\left(\omega_{0} \Delta t\right)^{2} .
$$

This analysis shows that this method is significantly less accurate that the method based on trapezoidal integration. The error in the real part of the frequency and one of the errors in the damping are both four times greater. Also, this method results in numerical damping of the uncoupled wall dynamics; this numerical damping of magnitude $\frac{1}{4}\left(\omega_{0} \Delta t\right)^{3}$ could be significant relative to the true physical damping when $d$ is $0.005-0.01$ and $\omega_{0} \Delta t$ is 0.1 or larger. 
This same criticism can be applied to many other methods frequently used for structural dynamics, including the Houboult, Wilson- $\theta$ and Newmark- $\beta$ methods, and the multi-parameter unified schemes of Zienkiewicz et al [15] and Thomas and Gladwell [12]. Some of these methods always introduce numerical damping; the others depend on a set of parameters which are often chosen to ensure some level of numerical damping. The reason that structural dynamicists prefer methods with numerical damping is that they are usually integrating very large stiff systems of equations in which some very high frequency modes are not adequately resolved by the chosen timestep. Therefore, (quoting from the paper by Thomas and Gladwell [12])

in practice we use methods which are damped ... since this ensures that the highly oscillatory eigenfunctions ... excited by noise in the initial data are damped out quickly.

In the application in this paper, there is only one structural eigenmode and so this concern does not arise. Furthermore, in a real 3D application it is assumed that a reduced modal representation of the structural dynamics would be used $[4,5,8,14]$, perhaps using the lowest five eigenmodes, and so again there would be no problem of numerical stiffness. If a very large number of structural eigenmodes are retained it may become desirable in implicit calculations to introduce structural damping into the equations for the highest frequency modes only, since these frequencies are unlikely to be adequately resolved by the large timestep. 


\subsection{State-transition algorithm}

An alternative algorithm for approximating the vector version of the wall dynamics equation is based on work by Edwards et al in the Unsteady Aerodynamics Branch at NASA Langley Research Center $[2,10]$. The objective is to construct an approximation which is exact when there is no aerodynamic coupling.

The exponential matrix $\exp (t A)$ is defined for an arbitrary matrix $A$ as

$$
\exp (t A)=\sum_{0}^{\infty} \frac{t^{n}}{n !} A^{n}
$$

By definition, $A^{0}$ is the identity matrix $I$ and so $\exp (t A)=I$ when $t=0$. Another important property of the exponential matrix is that

$$
\frac{d}{d t} \exp (t A)=A \exp (t A)=\exp (t A) A
$$

For the particular matrix $A$ in this analysis,

$$
\exp (t A)=\left(\begin{array}{rr}
\cos \left(\omega_{0} t\right) & -\sin \left(\omega_{0} t\right) \\
\sin \left(\omega_{0} t\right) & \cos \left(\omega_{0} t\right)
\end{array}\right) .
$$

This can be verified by checking that it satisfies the above two conditions, or by directly evaluating $A^{n}$ and using the series expansions for $\cos \left(\omega_{0} t\right)$ and $\sin \left(\omega_{0} t\right)$.

Using this matrix, it follows immediately that

$$
\frac{d}{d t}(\exp (t A) w)=\exp (t A) \frac{d w}{d t}+\exp (t A) A w=\exp (t A) P
$$

and hence

$$
w\left(t_{0}\right)-\exp \left(-t_{0} A\right) w(0)=\int_{0}^{t_{0}} \exp \left(\left(t-t_{0}\right) A\right) P(t) d t
$$

The state-transition method uses this equation with $t_{0}=\Delta t$ and a suitable approximation to the integral. The approximation used by Edwards et al is

$$
\int_{0}^{t_{0}} \exp \left(\left(t-t_{0}\right) A\right) P(t) d t \approx\left(\int_{0}^{t_{0}} \exp \left(\left(t-t_{0}\right) A\right) d t\right) \frac{1}{2}\left(P(0)+P\left(t_{0}\right)\right) .
$$

Although this is a second order accurate approximation to the integral, meaning that the relative error is $O\left(\Delta t^{2}\right)$, it is better for the model problem in this paper to use trapezoidal integration which is also second order accurate.

$$
\int_{0}^{t_{0}} \exp \left(\left(t-t_{0}\right) A\right) P(t) d t \approx \frac{t_{0}}{2}\left(\exp \left(-t_{0} A\right) P(0)+P\left(t_{0}\right)\right) .
$$

This gives the final algorithm,

$$
w^{(n+1)}=\exp (-\Delta t A)\left(w^{(n)}+\frac{1}{2} \Delta t P^{(n)}\right)+\frac{1}{2} \Delta t P^{(n+1)} .
$$


A predictor/corrector procedure can again be used to avoid the problems due to the implicit nature of this algorithm.

$$
\left.\begin{array}{rl}
P^{*} & =2 P^{(n)}-P^{(n-1)} \\
w^{*} & =\exp (-\Delta t A)\left(w^{(n)}+\frac{1}{2} \Delta t P^{(n)}\right)+\frac{1}{2} \Delta t P^{*}
\end{array}\right\} \text { Predictor }
$$

Again, the predictor/corrector combination gives results which are within $O\left(d^{2}\right)$ of those which would be obtained from the original implicit coupling. For simplicity, it is the implicit coupling which is now analysed for stability and accuracy.

Assuming that upwind differencing is used for the aerodynamic equations then $r_{j}^{(n)}=0$ for all $j$ and $n$, and hence

$$
P^{(n)}=\left(\begin{array}{c}
0 \\
-\frac{\rho c}{m} \dot{x}_{w}^{(n)}
\end{array}\right)=\left(\begin{array}{c}
0 \\
-2 d \omega_{0} \dot{x}_{w}^{(n)}
\end{array}\right) .
$$

Therefore, Equation (4.26) becomes

$$
\left(\begin{array}{cc}
1 & 0 \\
0 & 1+d \omega_{0} \Delta t
\end{array}\right) w^{(n+1)}=\left(\begin{array}{cc}
\cos \left(\omega_{0} \Delta t\right) & \sin \left(\omega_{0} \Delta t\right)\left(1-d \omega_{0} \Delta t\right) \\
-\sin \left(\omega_{0} \Delta t\right) & \cos \left(\omega_{0} \Delta t\right)\left(1-d \omega_{0} \Delta t\right)
\end{array}\right) w^{(n)}
$$

$w^{(n)}=z^{n} W$ is a solution for some non-trivial constant vector $W$ if and only if

$$
\begin{gathered}
\operatorname{det}\left(\begin{array}{rr}
z-\cos \left(\omega_{0} \Delta t\right) & -\sin \left(\omega_{0} \Delta t\right)\left(1-d \omega_{0} \Delta t\right) \\
\sin \left(\omega_{0} \Delta t\right) & z\left(1+d \omega_{0} \Delta t\right)-\cos \left(\omega_{0} \Delta t\right)\left(1-d \omega_{0} \Delta t\right)
\end{array}\right)=0 \\
\Longrightarrow \quad z^{2}\left(1+d \omega_{0} \Delta t\right)-2 z \cos \left(\omega_{0} \Delta t\right)+\left(1-d \omega_{0} \Delta t\right)=0 \\
\Longrightarrow \quad z=\frac{\cos \left(\omega_{0} \Delta t\right) \pm \sqrt{-\sin ^{2}\left(\omega_{0} \Delta t\right)+\left(d \omega_{0} \Delta t\right)^{2}}}{1+d \omega_{0} \Delta t}
\end{gathered}
$$

When $d=0, z=\cos \left(\omega_{0} \Delta t\right) \pm i \sin \left(\omega_{0} \Delta t\right)=\exp \left( \pm i \omega_{0} \Delta t\right)$, confirming that the algorithm gives the exact analytic solution because of the way in which it was constructed.

When $0<d \ll 1$, let $z=\exp (i \omega t)$, then provided $\sin \left(\omega_{0} \Delta t\right) \neq 0$ it follows that

$$
\begin{gathered}
\mathcal{R}(\omega)=\frac{\arg z}{\Delta t}=\omega_{0}\left(1-\frac{1}{2} d^{2} \frac{\omega_{0} \Delta t}{\tan \left(\omega_{0} \Delta t\right)}\right)+O\left(d^{4}\right) \\
\mathcal{I}(\omega)=\frac{\log \left|z^{-1}\right|}{\Delta t}=\frac{1}{\Delta t} \log \left(\frac{1+d \omega_{0} \Delta t}{\sqrt{1-\left(d \omega_{0} \Delta t\right)^{2}}}\right)=d \omega_{0}+\frac{1}{3} d \omega_{0}\left(d \omega_{0} \Delta t\right)^{2}+O\left(d^{5}\right),
\end{gathered}
$$




$$
\Longrightarrow \quad \frac{\omega}{\omega_{0}}=1+i d-\frac{1}{2} d^{2} \frac{\omega_{0} \Delta t}{\tan \left(\omega_{0} \Delta t\right)}+\frac{1}{3} i d\left(d \omega_{0} \Delta t\right)^{2}+O\left(d^{4}\right)
$$

Thus, the algorithm gives the exact analytic frequency to within $O\left(\left(d \omega_{0} \Delta t\right)^{2}\right)$ which is extremely small for $d \ll 1$. This is a surprisingly good result, which can be better understood by integrating the analytic o.d.e. over one period to obtain

$$
w\left(2 \pi / \omega_{0}\right)-w(0)=\int_{0}^{2 \pi / \omega_{0}} \exp (-t A) P(t) d t .
$$

The corresponding discrete solution replaces the integral by the trapezoidal integration approximation. When $d \ll 1$, to leading order $w(t)$ and hence $P(t)$ vary sinusoidally. $\exp (-t A)$ also varies sinusoidally and so the product has terms which are either constant or sinusoidal with frequency $2 \omega_{0}$. The trapezoidal integration of the constant is always exact, and the integration of the sinusoidal term correctly gives zero provided there is more than one timestep during the period $\pi / \omega_{0}$; this corresponds to the constraint $\sin \left(\omega_{0} \Delta t\right) \neq 0$.

The conclusion from this analysis is that the state-transition integration of the structural dynamics equations is very accurate even when there are relatively few timesteps per period. Since it provides both $x_{w}$ and $\dot{x}_{w}$ at each timestep it is also easy to construct highly accurate discretisations of the kinematic boundary condition. In this model 1D problem, the accuracy with which the aerodynamic equations are approximated does not affect the predicted aerodynamic damping of the wall's motion. In the real 3D application it would affect the result, so it is then the accuracy of the aerodynamic calculation which would determine the accuracy of the coupled aeroelastic analysis.

Following the slightly different discretisation used by Edwards et al,

$$
\int_{0}^{t_{0}} \exp \left(\left(t-t_{0}\right) A\right) d t=\frac{1}{\omega_{0}}\left(\begin{array}{rr}
\sin \left(\omega_{0} t_{0}\right) & 1-\cos \left(\omega_{0} t_{0}\right) \\
-\left(1-\cos \left(\omega_{0} t_{0}\right)\right) & \sin \left(\omega_{0} t_{0}\right)
\end{array}\right),
$$

and hence the modified equation for $z$ is

$$
\begin{gathered}
\operatorname{det}\left(\begin{array}{rr}
z-\cos \left(\omega_{0} \Delta t\right) & -\sin \left(\omega_{0} \Delta t\right)+d(z+1)\left(1-\cos \left(\omega_{0} \Delta t\right)\right. \\
\sin \left(\omega_{0} \Delta t\right) & z-\cos \left(\omega_{0} \Delta t\right)+d(z+1) \sin \left(\omega_{0} \Delta t\right)
\end{array}\right)=0 \\
\Longrightarrow \quad z^{2}\left(1+d \sin \left(\omega_{0} \Delta t\right)\right)-2 z \cos \left(\omega_{0} \Delta t\right)+\left(1-d \sin \left(\omega_{0} \Delta t\right)\right)=0 \\
\Longrightarrow \quad z=\frac{\cos \left(\omega_{0} \Delta t\right) \pm i \sin \left(\omega_{0} \Delta t\right) \sqrt{1-d^{2}}}{1+d \sin \left(\omega_{0} \Delta t\right)}
\end{gathered}
$$

When $d=0$ this leads to

$$
\frac{\omega}{\omega_{0}} \approx 1+i d \frac{\sin \left(\omega_{0} \Delta t\right)}{\omega_{0} \Delta t}-\frac{1}{2} d^{2} \cos \left(\omega_{0} \Delta t\right) \frac{\sin \left(\omega_{0} \Delta t\right)}{\omega_{0} \Delta t}+\frac{1}{3} i d\left(d \omega_{0} \Delta t\right)^{2}\left(\frac{\sin \left(\omega_{0} \Delta t\right)}{\omega_{0} \Delta t}\right)^{3}
$$


The $O(d)$ term is no longer obtained exactly independent of the value of $\omega \Delta t$, but the error is $O\left(d\left(\omega_{0} \Delta t\right)^{2}\right)$ which is very small if $\omega_{0} \Delta t<0.1$. In practice, for $3 \mathrm{D}$ computations this method is probably as accurate as the other discretisation of the state-transition equations, since in both cases the main error will come from the discretisation of the fluid equations.

A final remark is that there are two ways in which to handle weak structural damping and weakly nonlinear structural dynamics. The first is to decompose $A$ into a dominant part which is constant in time and has purely imaginary eigenvalues, plus a smaller part which contains the damping terms and nonlinear stiffness terms; because of the nonlinear dependence on $x_{w}$ and $\dot{x}_{w}$ this smaller part will vary in time.

$$
A(t)=A_{0}+A_{1}(t) .
$$

The wall dynamic equation can be written as

$$
\frac{d w}{d t}+A_{0} w=\left(P-A_{1} w\right)
$$

and hence

$$
w\left(t_{0}\right)-\exp \left(-t_{0} A_{0}\right) w(0)=\int_{0}^{t_{0}} \exp \left(\left(t-t_{0}\right) A_{0}\right)\left(P(t)-A_{1}(t) w(t)\right) d t .
$$

The relatively small extra term $A_{1}(t) w(t)$ can then be approximated in exactly the same manner as the aerodynamic term, using trapezoidal integration and a predictor/corrector solution procedure.

The alternative is to define

$$
P(t)=P^{(n)}+\frac{t-t^{(n)}}{\Delta t}\left(P^{(n+1)}-P^{(n)}\right),
$$

for the interval $t^{(n)} \leq t \leq t^{(n+1)}$, and then solve the o.d.e.

$$
\frac{d w}{d t}+A w=P(t)
$$

with initial conditions at $t^{(n)}$ to obtain the solution at $t^{(n+1)}$. This can be done using any numerical algorithm with sufficiently small timesteps to achieve any desired level of accuracy. Even if there are many structural timesteps for each aerodynamic timestep the computational cost will be negligible since there will be very few structural modes compared to the number of aerodynamic grid points. 


\section{Discussion of relevance to $3 \mathrm{D}$ applications}

The interpretation of the analysis in this paper in the context of real 3D engineering calculations is a tricky issue. The simple model problem in the current analysis has a one-degree-of-freedom structural oscillation in which the surface pressure varies in phase with the wall's velocity, causing aerodynamic damping under all conditions. In a real application the structural model will have sev-

eral degrees of freedom. For each degree of freedom there is a corresponding generalised force which is the combined effect of the entire surface pressure distribution on the particular mode of vibration. The nondimensional generalised force will have magnitude corresponding to the damping factor $d$ in the model problem, but unlike the model problem the generalised force will not be perfectly in phase with the mode's motion. Flutter, a physical instability of the coupled aeroelastic system, occurs when the phase difference between the force and the velocity of the mode is greater than $90^{\circ}$. This corresponds to redefining the damping factor $d$ in the model analysis to be a complex quantity with negative real component. For accurate prediction of the conditions under which flutter occurs, it is therefore the phase rather than the magnitude of the aerodynamics which must be accurately captured by the numerical discretisation. Treating $d$ as a general complex quantity, it can be seen that the analyses of the first order coupling with both the upwind and Lax-Wendroff discretisations show significant phase errors unless the timestep is very small. The analysis of the second order coupling shows the leading error is in the magnitude of the aerodynamic effect; there is only a third-order error in its phase. These methods are therefore very much more accurate. 


\section{Conclusions}

The principal conclusions from this study are:

- The key non-dimensional physical parameter in the model problem is the aerodynamic damping factor $d$. For turbomachinery applications this lies in the range $0.005-0.02$; for aircraft applications it is usually in the range 0.05-0.2. To simplify the analysis in this study, asymptotic approximations are made on the basis that $d \ll 1$. For larger values of $d$ numerical computation would be required to obtain the complex frequencies arising from the numerical discretisation.

- The other important non-dimensional parameter in this analysis is the product of the natural frequency of the wall's vibration and the timestep, $\omega_{0} \Delta t$, which is inversely proportional to the number of timesteps per period.

- There appears to be no possibility of a spurious numerical instability due to the coupling of the aerodynamic and structural models, provided there are no unstable or neutrally stable spurious modes in the uncoupled limit as $d \rightarrow 0$.

- If an explicit CFD algorithm is used for the aerodynamic equations, then for typical flutter frequencies and aerodynamic grid resolution $\omega_{0} \Delta t$ will be $O\left(10^{-3}\right)$. Hence, any stable algorithm for the discretisation of the structural dynamics and the kinematic boundary condition will be sufficiently accurate provided it is at least second order accurate for the uncoupled vibration, and first order accurate for the coupled analysis.

- If an implicit CFD algorithm is used for the aerodynamic equations, then it is possible that $\omega_{0} \Delta t$ will be $O\left(10^{-1}\right)$. In this case it is necessary to use a discretisation which is second-order accurate for both the uncoupled and coupled systems. It is also best to avoid the use of the many standard algorithms which cause spurious numerical damping of the uncoupled wall dynamics; these methods may be very suitable for extremely stiff systems but are not suitable for this application in which there is very little physical damping and its accurate evaluation is essential.

- The state-transition structural algorithm has excellent numerical properties which make it particularly suitable for calculations with extremely few timesteps per period. Trapezoidal integration of the vector form of the wall dynamic equation is also very accurate.

- By using the analogy between complex values of $d$ in the model problem and real 3D engineering applications, it is thought that the analysis resolves many of the central issues in the stability and accuracy of coupled discretisations of aeroelastic systems. 


\section{References}

[1] J. Alonso, L. Martinelli, and A. Jameson. Multigrid unsteady Navier-Stokes calcultations with aeroelastic applications. AIAA Paper 95-0048, 1995.

[2] J.W. Edwards, R.M. Bennett, W. Whitlow, and D.A. Seidel. Time-marching transonic flutter solutions including angle-of-attack effects. Journal of Aircraft, 20(11):899-906, 1983.

[3] S.K. Godunov and V.S. Ryabenkii. The Theory of Difference Schemes-An Introduction. North Holland, Amsterdam, 1964.

[4] G.P. Guruswamy. ENSAERO - a multidisciplinary program for fluid/structural interaction studies of aerospace vehicles. Computing Systems in Engineering, 1(2-4):237-256, 1990.

[5] G.P. Guruswamy. Unsteady aerodynamic and aeroelastic calculations for wings using Euler equations. Journal of the American Institute on Aeronautics and Astronautics, 28(3), 1990.

[6] B. Gustafsson, H.-O. Kreiss, and A. Sundström. Stability theory of difference approximations for mixed initial boundary value problems. II. Mathematics of Computation, 26(119):649-686, Jul 1972.

[7] A. Jameson. Time dependent calculations using multigrid with applications to unsteady flows past airfoils, wings and rotors. AIAA Paper 91-1596, 1991.

[8] J.G. Marshall and M. Imregun. A 3D time-domain flutter prediction method for turbomachinery blades. In Proc. Int. Forum on Aeroelasticity and Structural Dynamics, Royal Aeronautical Society, Manchester, 1995.

[9] T. Nomura and T.J.R. Hughes. An arbitrary Lagrangian-Eulerian finite element method for interaction of fluid and a rigid body. Computer Methods in Applied Mechanics and Engineering, 95:115-138, 1992.

[10] R.D. Rausch, J.T. Batina, and H.T.Y. Yang. Three-dimensional timemarching aeroelastic analyses using an unstructured-grid Euler method. Journal of the American Institute on Aeronautics and Astronautics, 31(9):1626-1633, 1993.

[11] R.D. Richtmyer and K.W. Morton. Difference Methods for Initial-Value Problems. Wiley-Interscience, 2nd edition, 1967. Reprint edn (1994) Krieger Publishing Company, Malabar.

[12] R. Thomas and I. Gladwell. Variable-order variable-step algorithms for second-order systems. Part I: the methods. International Journal for $\mathrm{Nu}$ merical Methods in Engineering, 26:39-53, 1988. 
[13] L.N. Trefethen. Group velocity interpretation of the stability theory of Gustafsson, Kreiss, and Sundström. Journal of Computational Physics, 49:199-217, 1983.

[14] B. Winzell. Recent applications of linear and nonlinear unsteady aerodynamics for aeroelastic analysis. AGARD CP 506, Oct 1991.

[15] O. Zienkiewicz, W. Wood, N. Hine, and R. Taylor. A unified set of single step algorithms. Part I: general formulation and applications. International Journal for Numerical Methods in Engineering, 20:1529-1552, 1984. 